lar branches of the tree of learning. If highly specialized books such as this are to be useful, a more serious attempt has to be made to cut through the jargon and establish stronger lines of communication.

The content of the book ranges from the smell of histocompatibility antigens (this is a joke not a paradox, if all that has been written about histocompatibility antigens relates to an outbreeding system rather than say associative recognition at the cellular level), through the lack of class I type histocompatibility antigen polymorphisms in Syrian hamsters (how can they survive when such polymorphisms are deemed to be almost a main staff of life), to the enhancement of skin allografts by prior blood transfusion (it should immunize). The astonishing fact that many tumours can remain dormant for years is seen as a contradiction of the notion that the immune response is designed in part to see tumours off. I am inclined to agree with the commentator (Richmond Prehn) who observes laconically "the fact of dormancy constitutes a strong argument against the thesis that the immune response is the major homeostatic mechanism vis à vis cancer". In other words, the apparent paradox strikes at the root of the working hypothesis, which clear indication is, in turn (and paradoxically) largely ignored by the authors of the chapter in question.

Many of the chapters are concerned with the paradigm of the immune response as a defence mechanism, which indeed it may be. But interested immunologists should pay attention to at least the title of a paper quoted on p.114 ("Thomas, L. 'Symbiosis as an immunologic problem' in The Immune System and Infectious Diseases, Milgrom, F. and Neter, E., eds, S. Karger, Basel, 1975, 2 "). If in fact the immune response is not by design rejectional but accommodatory, then at least a proportion of the paradoxes vanish overnight - but that's my prejudice!

The book merits careful reading. It illustrates the ease with which immunologists remain encapsulated in the intellectual straitjackets that they themselves have put on, and the almost catechismal rigidity with which they regard working hypotheses. If the editors were to publish one of these paradoxes a month in some more popular and less-expensive conveyance of scientific news, if they were to insist both in advance and by editing that the exact nature of the apparent paradoxes was intelligibly displayed, if they were to read again the fable of the Emperor's clothes, then they just could produce something of value in these uneasy days of grant-supported science. $\square$

Tony Davies is Professor of Immunobiology and Deputy Director at the Institute of Cancer Research, Haddow Laboratories, Clifton Ave. nue, Belmont Sutton, Surrey SM2 5PX, UK.

\section{Empirical insight}

\section{Peter E. Hodgson}

The Neglect of Experiment. By Allan Franklin. Cambridge University Press: 1987. Pp.290. £30, $\$ 42.50$.

FEw philosophers of science have spent their formative years struggling with recalcitrant apparatus and trying to decide whether some observations really are significant or should be attributed to statistical fluctuations or equipment malfunction. Most of them started as theoretical physicists or mathematicians before they turned to philosophy, and so tend to see the history of science as the battleground of competing theories, with only occasional reference to experiments. Such an approach ignores a whole series of questions bearing on the understanding of science: Why do scientists decide to do a particular experiment? How do they know when the results are significant? Why are some anomalies ignored and others hailed as decisive discoveries?

Professor Franklin began as an experimental high-energy physicist and so has practical experience of these matters. He now works on the history and philosophy of science, and so is well-equipped to redress the balance. Problems about the role of experiment have been discussed by philosophers, but usually in the abstract. Franklin adopts the empirical approach, analysing in detail several decisive experiments in elementary particle physics. He has uncovered a wealth of fascinating detail that supports the validity of the methods used and shows the inadequacy of many philosophical accounts. As far as possible, he has used the original data, the laboratory notebooks and the personal recollections of the scientists who did the work concerned.

His first example of a crucial experiment is that leading to the discovery of parity non-conservation. The experimental evidence was overwhelming; it solved the $\tau-\theta$ puzzle and is of vital importance in elementary particle and nuclear physics. Subsequently it was confirmed by a wide range of different experiments. Oddly enough, convincing results of parity-violating experiments were published in 1928 and 1930, but the implications were not recognized. Franklin suggests that this was partly because of the lack of an appropriate theoretical context at the time, and partly because the issues at stake were overshadowed by other contemporary arguments.

His next example is the experiment of Cronin, Fitch and colleagues that refuted the concept of the invariance of physical processes under combined space inversion and particle-antiparticle interchange. This was a complicated experiment that required exhaustive tests of all conceivable alternative hypotheses. Not all of these are reported in the original papers, which therefore tend to give the unwary reader an inadequate impression of the validity of the procedure.

A simpler example is provided by Millikan's oil-drop experiment that established the quantization of charge and gave an accurate value for the charge of the electron. Franklin examined Millikan's notebooks and repeated his calculations. He found that although Millikan said that he had published all his results, he had in fact been somewhat selective. One series of measurements did not give the "expected"

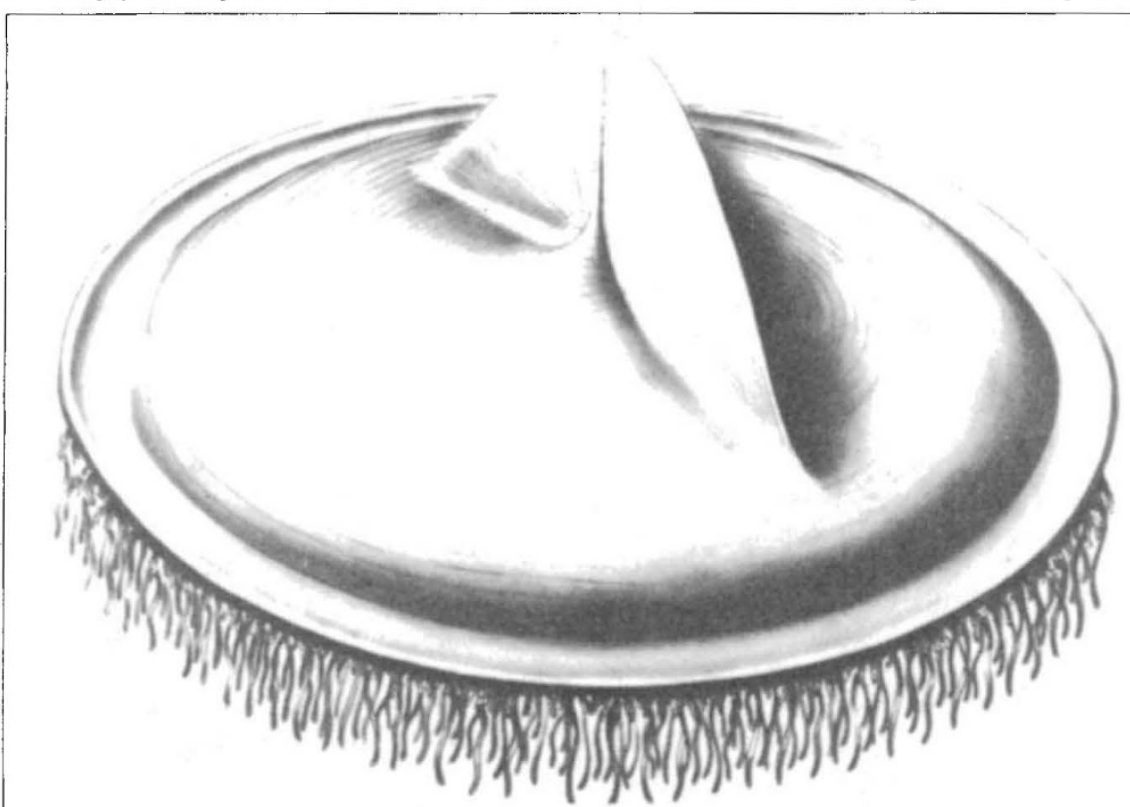

Sailing back - reconstruction of the palaeozoic hydrozoan Plectodiscus discoideus. The picture is taken from Problematic Fossil Taxa, edited by Antoni Hoffman and Matthew H. Nitecki and published by Oxford University Prcss. Price is $£ 60, \$ 69$. 